\title{
Magnetic-Field Induced Slow Relaxation in the Ising-Like Quasi-One-Dimensional Ferromagnet $\mathrm{KEr}\left(\mathrm{MoO}_{4}\right)_{2}$
}

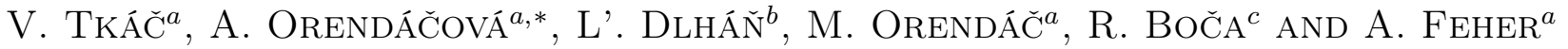 \\ ${ }^{a}$ Centre of Low Temperature Physics of SAS and P.J. Šafárik University, \\ Park Angelinum 9, 04001 Košice, Slovak Republic \\ ${ }^{b}$ Institute of Inorganic Chemistry, FCHPT, Slovak University of Technology, 81237 Bratislava, Slovakia \\ ${ }^{c}$ Department of Chemistry, FPV, University of SS Cyril and Methodius, 91701 Trnava, Slovakia
}

\begin{abstract}
We present the study of spin dynamics of $\operatorname{KEr}\left(\mathrm{MoO}_{4}\right)_{2}$ in the magnetic field applied along the hard axis $c$. The temperature dependence of AC susceptibility in zero magnetic field studied at frequencies $f=10,100$, and $1000 \mathrm{~Hz}$ indicated the absence of relaxation in the temperature range from 2 to $20 \mathrm{~K}$. Application of magnetic field induced a slow magnetic relaxation, which was investigated in detail in the field $0.5 \mathrm{~T}$. The highest intensity of the relaxation process, reflected by the values of imaginary susceptibility was observed at $2 \mathrm{~K}$. With increasing temperature, the relaxation process is weaker and vanishes completely above $3.5 \mathrm{~K}$. Corresponding Cole-Cole diagrams were constructed and analyzed within a single relaxation process which can be associated with a direct relaxation process with a bottleneck effect, $\tau \approx 1 / T^{b}$, and $b=1.4$. The slow relaxation at $2 \mathrm{~K}$ intensifies with increasing magnetic field at least up to $1 \mathrm{~T}$.
\end{abstract}

DOI: 10.12693/APhysPolA.131.893

PACS/topics: 76.60.Es, 76.30.Kg, 75.30.Gw, 75.40.Gb

\section{Introduction}

Materials exhibiting magnetocaloric effect (MCE), in which a temperature and entropy change, $\Delta S$, occurs in response to a change of magnetic field, have gained increasing interest for solid state magnetic cooling. Besides room-temperature applications [1], large MCE in low-temperature region can be utilized, e.g. in the process of liquefaction of helium for cryogenics, or hydrogen in fuel industry [2]. At low temperatures, the lattice specific heat decreases, consequently, more options for large MCE are available and besides the systems with the first and second-order phase transitions, also paramagnets and superparamagnets can be employed. Concerning the realization of $\mathrm{MCE}$, the magnetic cooling can be achieved by sweeping magnetic field. Another way is the use of strongly anisotropic refrigerants, in which the large MCE can be achieved by simply rotating single crystal in a constant magnetic field [3].

Recent studies of magnetocaloric properties of a single crystal of $\mathrm{KEr}\left(\mathrm{MoO}_{4}\right)_{2}$ revealed large conventional MCE around $10 \mathrm{~K}\left(-\Delta S_{\max }=14 \mathrm{~J} /(\mathrm{kg} \mathrm{K})\right.$ for $\left.5 \mathrm{~T}\right)$ in the field applied along the easy axis $a$. A huge anisotropy of MCE was observed in the $a b$ plane with $-\Delta S_{r}, \max =10$ and $13 \mathrm{~J} /(\mathrm{kg} \mathrm{K})$ achieved by a simple rotating of the single crystal within the $a b$ plane in the constant magnetic field 2 and $5 \mathrm{~T}$, respectively [4]. Thus, $\mathrm{KEr}\left(\mathrm{MoO}_{4}\right)_{2}$ may serve as a promising candidate for the implementation of a compact rotary magnetic cryorefrigerator.

*corresponding author; e-mail: alzbeta.orendacova@upjs.sk
In this respect, the study of time dependence and rate effects of the MCE is relevant for the use of $\mathrm{KEr}\left(\mathrm{MoO}_{4}\right)_{2}$ as a practical magnetic refrigerant with reasonable operation frequencies. Previous investigation of magnetic relaxation in this material was performed in the magnetic field applied along the easy axis and a field-induced slow relaxation occurred in the wide temperature range spanning from 2 to $20 \mathrm{~K}$ [5]. Development of imaginary component of $\mathrm{AC}$ susceptibility at $2 \mathrm{~K}$ was studied in magnetic field spanning from 0 to $1 \mathrm{~T}$ and the highest values appeared in fields around $0.2-0.3 \mathrm{~T}$. The fields correspond to the region of a maximum increase of isothermal magnetization at $2 \mathrm{~K}[5]$.

Previous studies revealed anomalous behavior of isothermal magnetization at temperatures between 2 and $3 \mathrm{~K}$ in the field applied along the $c$-axis [6]. At higher temperatures the anomaly vanished, while at lower temperatures, the anomaly developed to a well defined plateau between 0.5 and $1 \mathrm{~T}$ [7]. Present work focuses at the investigation of relaxation effects in the region of the anomaly.

\section{Experimental details}

The $\operatorname{KEr}\left(\mathrm{MoO}_{4}\right)_{2}$ is a pink, transparent and soft material that was prepared by a standard flux method at the B. Verkin Institute of Low Temperature Physics and Engineering in Kharkov. The compound is a magnetic insulator with a layered crystal structure and crystallizes in the orthorhombic space group $P b c n\left(D_{2 h}^{14}\right)[6]$. The first coordination sphere of $\mathrm{Er}^{3+}$ consists of eight oxygen atoms forming a slightly distorted square antiprism. The ground state of a free $\mathrm{Er}^{3+}$ is ${ }^{4} I_{15 / 2}$ multiplet, which is split into 8 Kramers doublets with the energies $E_{1}=22$, 
$E_{2}=46, E_{3}=107, E_{4}=266, E_{5}=372, E_{6}=456$, and $E_{7}=467 \mathrm{~K}$ above the ground doublet [4]. Interplay of crystal field and dipolar interactions results in the formation of a two-dimensional array of the Ising ferromagnetic chains below $2 \mathrm{~K}$. The antiferromagnetic interchain coupling leads to the phase transition to magnetic long-range order at $0.9 \mathrm{~K}[8]$.

AC susceptibility was investigated in the temperature range from $1.8 \mathrm{~K}$ to $20 \mathrm{~K}$ in magnetic fields up to $5 \mathrm{~T}$ and frequencies from $0.1 \mathrm{~Hz}$ up to nominally $1 \mathrm{kHz}$ in a commercial Quantum Design SQUID magnetometer. AC component of alternating field had an amplitude $0.25 \mathrm{mT}$ and was applied along the $c$-axis. A single crystal with a mass of $12 \mathrm{mg}$ was used for these magnetic measurements.

\section{Results and discussion}

Temperature dependence of AC susceptibility in zero magnetic field was studied at the frequencies 10, 100, and $1000 \mathrm{~Hz}$ (Fig. 1, inset).

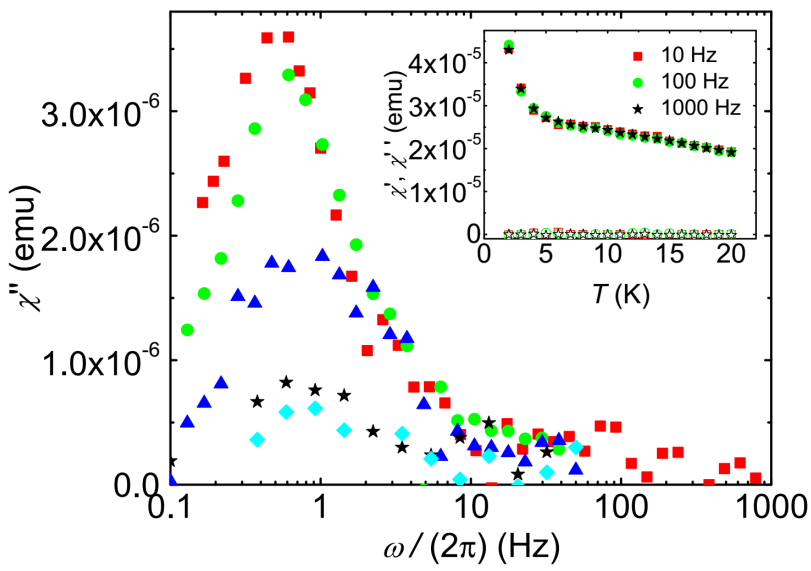

Fig. 1. Frequency dependence of imaginary component of AC susceptibility of $\operatorname{KEr}\left(\mathrm{MoO}_{4}\right)_{2}$ in magnetic field $0.5 \mathrm{~T}$. Squares, circles, triangles, stars, and diamonds represent data at constant temperatures $2,2.25$, $2.75,3.25$, and $3.5 \mathrm{~K}$, respectively. Inset: temperature dependence of real (full symbols) and imaginary (open symbols) component of AC susceptibility in zero magnetic field.

In the studied frequency range, the real component, $\chi^{\prime}$, does not depend on the frequency and corresponds to DC susceptibility while the imaginary component, $\chi^{\prime \prime}$, is zero. Application of magnetic field $0.5 \mathrm{~T}$ introduces slowing down of the relaxation reflected in the nonzero values of $\chi^{\prime \prime}$ (Fig. 1). The frequency dependence of $\chi^{\prime \prime}$ is characterized by a round maximum which grows with decrease of temperature and shifts to lower frequencies. At higher temperatures above $3.5 \mathrm{~K}$ the maximum vanishes in the noise. Corresponding Cole-Cole diagrams (Fig. 2) were fitted by the Cole-Cole equation for a single relaxation process [9]:

$$
\chi(\omega)=\chi_{S}+\frac{\chi_{T}-\chi_{S}}{1+(\mathrm{i} \omega \tau)^{1-\alpha}} .
$$

$\chi_{T}$ and $\chi_{S}$ denote isothermal and adiabatic susceptibility, respectively, parameter $\alpha$ describes distribution of relaxation times; infinite distribution corresponds to $\alpha=1$ and a single relaxation time is represented by $\alpha=0$. Despite rather high level of noise, parameter $\alpha$ remains zero in the whole studied temperature range, indicating only one slow relaxation process at the time scale $0.1 \mathrm{~s}$ exists and vanishes at higher temperatures. Development of the relaxation time, $\tau$, with temperature is depicted in Fig. 3.

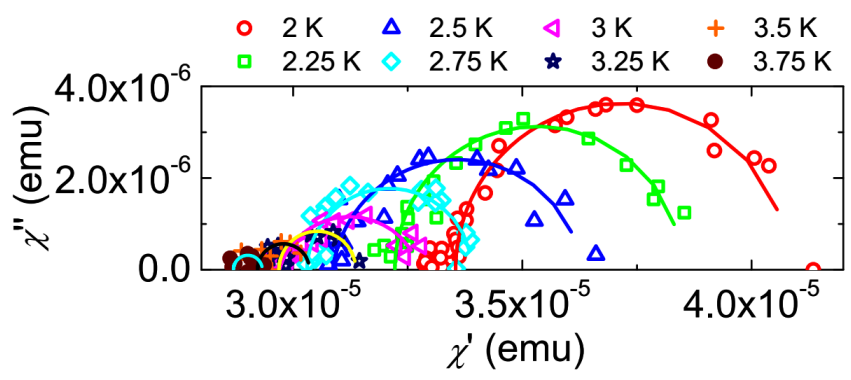

Fig. 2. Cole-Cole diagrams in the field $0.5 \mathrm{~T}, B \| c$, at various constant temperatures. The solid lines represent fits with Eq. (1).

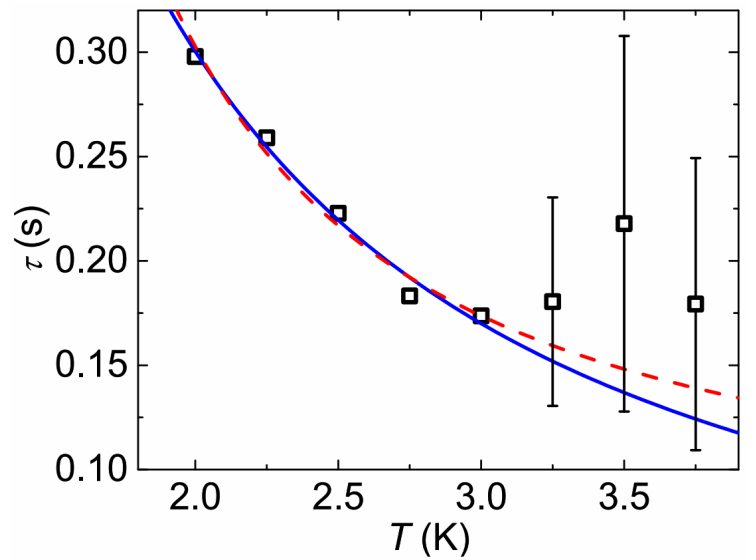

Fig. 3. Temperature dependence of relaxation time in the field $0.5 \mathrm{~T}, B \| c$. Dashed and solid lines represent fit by the Arrhenius equation and direct process with a bottleneck effect, respectively.

Since the available temperature range where the relaxation can be observed is rather narrow, it was not so easy to distinct between different temperature dependences of $\tau$.

In the first step, the data were fitted by a standard Arrhenius equation describing thermally activated relaxation process

$$
\tau=\tau_{0} \exp \left(\Delta / k_{\mathrm{B}} T\right)
$$

with $\tau_{0}=0.06 \mathrm{~s}$ and $\Delta / k_{\mathrm{B}}=3.3 \mathrm{~K}$. The large value of 
$\tau_{0}$ indicates a presence of a "phonon bottleneck (PB)" effect. In the case of the Orbach process, which can be also described by Eq. (2), the energy barrier, $\Delta$, corresponds to the energy difference between the ground and excited doublet. Since the gap in $\mathrm{KEr}\left(\mathrm{MoO}_{4}\right)_{2}$ is about $22 \mathrm{~K}$, at studied temperatures, the occupancy of the higher doublet can be neglected and the main contribution to the relaxation can be assigned to a direct process within the ground doublet. Considering the presence of PB effect and the direct process, the data were fitted with the relation [10]:

$$
\tau=a / T^{b} \text {. }
$$

The parameter $b$ changes from 1 for the absence of $\mathrm{PB}$ effect and 2 with dominant $\mathrm{PB}$ effect. The fitting procedure yielded $a=0.8 \mathrm{~s} \mathrm{~K}^{b}$ and $b=1.4$, which indicates the presence of the $\mathrm{PB}$ effect caused by a massive production of hot phonons with the energy equal to the splitting of the ground doublet in the magnetic field. It is evident that the hot phonons equilibrate with the phonon reservoir at higher temperatures above $3 \mathrm{~K}$ at the time scale outside of our experimental window.

To investigate the effect of magnetic field on the magnetic relaxation in more detail, temperature $2 \mathrm{~K}$ was chosen, where the relaxation is the most intensive. Thus, frequency dependence of AC susceptibility was investigated in magnetic fields lower and higher than $0.5 \mathrm{~T}$ (Fig. 4).

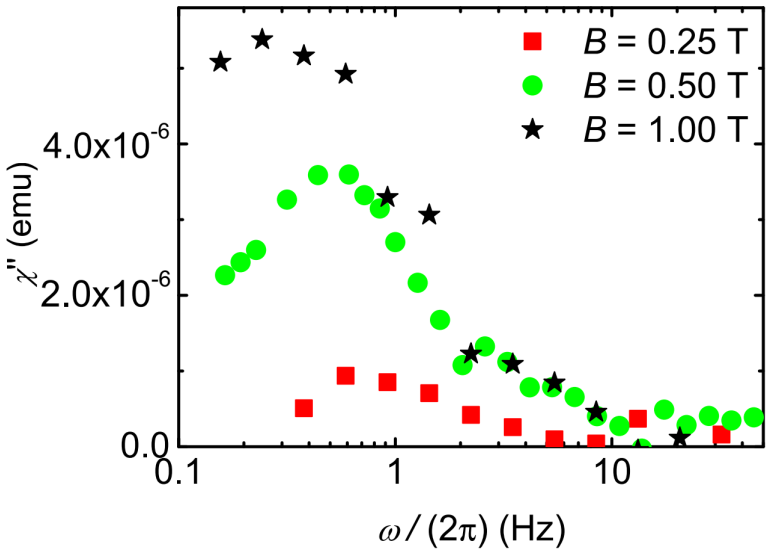

Fig. 4. Frequency dependence of imaginary component of AC susceptibility at $T=2 \mathrm{~K}$ in selected magnetic fields.

It can be seen that in comparison with $B=0.5 \mathrm{~T}$, the relaxation in the field $0.25 \mathrm{~T}$ is very weak and the maximum is shifted to higher frequencies. On the other hand, the relaxation in the field $1 \mathrm{~T}$ is pronounced and shifted to lower frequencies. The tendency suggests slowing down of the relaxation with increasing magnetic field. However, the tendency corrupts at higher magnetic fields $(2,3,4,5 \mathrm{~T})$ where $\chi^{\prime \prime}$ is zero within a noise and $\chi^{\prime}$ is frequency independent and coincides with the derivative of DC magnetization $\mathrm{d} M / \mathrm{d} B$. Such a behavior indicates a high rate of the energy exchange between the lattice and magnetic moments due to strong spin-phonon cou- pling [11]. To verify the conjecture, in future investigation of heat transport would be desirable.

\section{Conclusions}

A field induced magnetic relaxation in the field $0.5 \mathrm{~T}$ was observed at temperatures from 2 to $3.5 \mathrm{~K}$. At higher temperatures, the relaxation vanishes within our experimental frequency range. The analysis of relaxation time extracted from the fitting of the Cole-Cole diagrams indicated one relaxation process realized predominantly within the ground doublet. The relaxation mechanism has features of PB effect and direct process which intensifies at the field $1 \mathrm{~T}$. Higher fields than $1 \mathrm{~T}$ accelerate the relaxation while lower fields slow down the relaxation process. In future, thermal conductivity in magnetic field will be performed as well as AC susceptibility studies at temperatures below $2 \mathrm{~K}$ to investigate the slow relaxation in detail, where the effect of quantum tunneling and dipolar interactions can be expected.

\section{Acknowledgments}

This work was supported by the projects VEGA 1/0269/17, APVV-14-0078, APVV-14-0073 and ITMS 26220120047, 26220120005. Material support from U.S. Steel DZ Energetika is gratefully acknowledged.

\section{References}

[1] K.A. Gschneidner Jr., V.K. Pecharsky, A.O. Tsokol, Rep. Prog. Phys. 68, 1479 (2005).

[2] A. Midya, S.N. Das, P. Mandal, S. Pandya, V. Ganesan, Phys. Rev. B 84, 235127 (2011).

[3] M. Balli, S. Jandl, P. Fournier, M.M. Gospodinov, Appl. Phys. Lett. 104, 232402 (2014).

[4] V. Tkáč, A. Orendáčová, E. Čižmár, M. Orendáč, A. Feher, A.G. Anders, Phys. Rev. B 92, 024406 (2015).

[5] V. Tkáč, A. Orendáčová, R. Tarasenko, D.M. Pajerowski, E. Čižmár, M. Orendáč, A.G. Anders, M.W. Meisel, A. Feher, Acta Phys. Pol. A 127, 353 (2015).

[6] S. Mat’aš, E. Dudzik, R. Feyerherm, S. Gerischer, S. Klemke, K. Prokeš, A. Orendáčová, Phys. Rev. B 82, 184427 (2010).

[7] V. Tkáč, Ph.D. Thesis, P.J. Šafárik University, Košice 2014.

[8] D. Horváth, A. Orendáčová, M. Orendáč, M. Jaščur, B. Brutovský, A. Feher, Phys. Rev. B 60, 1167 (1999).

[9] K.S. Cole, R.H. Cole, J. Chem. Phys. 9, 341 (1941).

[10] P.L. Scott, C.D. Jeffries, Phys. Rev. 127, 32 (1962).

[11] V.I. Kutko, Low Temp. Phys. 31, 1 (2005). 